\title{
Grand Challenges in Wearable Electronics
}

\author{
Edward Sazonov ${ }^{1 *}$ and Walid A. Daoud ${ }^{2 *}$ \\ ${ }^{1}$ Department of Electrical and Computer Engineering, The University of Alabama, Tuscaloosa, AL, United States, ${ }^{2}$ School of \\ Energy and Environment, City University of Hong Kong, Kowloon, Hong Kong
}

Keywords: wearable electronics, sensors, on-body communications, flexible and stretchable electronics, electronic textiles, biodegradable electronics, batteries and energy harvesting

\section{OVERVIEW}

Wearable technology has deep roots in history, with eyeglasses and wrist and pocket watches being two of the most prolific technologies still being in use today. These technologies developed and evolved over the centuries. For example, watches started as decorative pieces based on an oscillating wheel and gear mechanism, later acquiring a primitive form of energy harvesting (selfwinding), progressing to electromechanical and then to fully electronic devices (McCrossen, 2013). Future watches may integrate sophisticated electronics, solar or thermal energy harvesting, carry a huge variety of functions beyond timekeeping and still be a decoration and a fashion statement. Eyeglasses is another vivid example, starting as assistive technology (Letocha and Dreyfus, 2002), also evolving to become a fashion accessory, frequently used by individuals who do not need vision correction. Eyeglasses are rapidly becoming a high-tech electronic device, with Google Glass, Alexa Frames, and the likes paving the way to everyday use of augmented reality. The long-term success of these two technologies is based on their utility, deep integration with daily activities, and social acceptance by the population (Degerli and Ozkan Yildirim, 2020). These examples are very valuable when we consider the challenges facing wearable electronics today.

Future growth in wearable devices is tightly coupled with advances in semiconductor technology and electronics. Wearables integrate a power source, sensors, data processing and storage core, actuators and displays, and a communication interface, packaged in a small, lightweight device that is worn on the body or integrated with everyday wear items, such as clothing, footwear, or accessories. Such wearables exemplify the cutting edge of the electronics, demanding the highest performance for the least amount of power, in the least amount of space, and in a challenging operating environment. Here wearables may gain a valuable lesson from a recent success storythe emergence of the smartphone as a ubiquitous communication device. The demands of modern smartphones closely match those of wearable devices, albeit in a larger package. The driving force behind the innovation and miniaturization of smartphones is high acceptability and demand across different cultures, generations, and societies. Resonating with the success of watches and eyeglasses, smartphones have a great utility that is tightly integrated into our life. Wearables may just as well become the next success story if they find an application that is demanded by the masses and if the electronic technology can support the effortless integration of wearables into the daily routine.

Looking into the electronic technologies that form the foundation of wearable devices, we can highlight several distinct areas:

Received: 16 February 2021 Accepted: 24 March 2021 Published: 27 April 2021

Citation: Sazonov E and Daoud WA (2021)

- Edge and Fog Computing (Bonomi et al., 2012; Shi et al., 2016): First and foremost, a wearable device is a computer with real-time processing capabilities, operating in extremely power-restricted environment. Modern wearables wield hefty computing power to execute signal processing and machine learning algorithms to process the information provided by the sensors (Witt et al., 2019). The advances in low-power digital electronics drive the capabilities of wearables. 
- Sensing: Sensors are the eyes and ears of wearable devices, measuring mechanical, electrical, chemical, optical, and other signals originating from the body and thus largely defining the utility available from a given device (Bandodkar and Wang, 2014; Nag et al., 2017; Ahmad Tarar et al., 2020; Shi et al., 2020). In the area of sensors, most of the recent progress is driven by advances in Micro-Electro-Mechanical Systems (MEMS) and Nanotechnologies (Jayathilaka et al., 2019).

- Storage: The wearables generate huge amounts of data, especially if raw sensor signals are required for a given application or communication capability is limited. Advances in solid-state storage are propelling the utilization of wearables in the collection of versatile, large datasets (Rajan et al., 2018).

- Communications and Internet of Things: In today's connected world, device-to-device communication is mandatory in the data processing pipeline. A wearable may be one of the many sources of information that are integrated into the "cloud" (Atzori et al., 2010). The expectation for a future wearable is that it is capable of communicating with other electronic devices, such as smartphones and tablets, where the human body itself may be considered as the communication medium to exchange information with other wearables. Progress in wireless and human body communications methods and related electronic devices will be continuously demanded (Cotton et al., 2014).

- Displays and actuators: Wearables may involve a variety of modalities in interfacing with the user, including tactile, haptic, visual, and audible information exchange (Pacchierotti et al., 2017; Gonçalves et al., 2018). These could be implemented as electronic or MEMS devices, facing the challenge of limited size and restricted power consumption.

- Battery technology and energy harvesting: Availability of power is the single, most important limiting factor in modern wearables. Developments in battery technology, electronic devices for efficient power conversion, and energy harvesting enable longer operation and richer functionality (Yang and Daoud, 2016; Kim J. et al., 2017; Dubal et al., 2018; Chong et al., 2019; Wang and Daoud, 2019a).

The technological advances in these areas were critical for the initial proliferation of wearable devices. The future of wearables will depend on the continuing progress and development of new solutions that provide high performance under power and space constraints.

\section{GRAND CHALLENGES}

Wearable devices and electronics present a unique interface of technology and humanity, thus producing unique challenges that need to account both for technological and human aspects of the problem. Human behavior may affect the operation of wearable as much as the technology advance. The following aspects may be considered as some of the challenges facing the field of wearables:

(a) Break-through applications of wearable electronics. From the dawn of history, the evolution of wearables is driven by the practicality, utility, and convenience they provide. The challenge of modern wearable electronics in discovering ubiquitous applications, as its future growth is contingent on emerging applications in health, wellness, and other personal needs. Novel applications of wearables may need to be supported by extensions of sensing and data analytics capabilities, thus presenting a compelling use case (Patel et al., 2012; Mukhopadhyay, 2015; Ahn et al., 2019; Degerli and Ozkan Yildirim, 2020).

(b) Minimization of user burden and integration with everyday wear items. The illustrations of wearable devices frequently include pictures of individuals instrumented at every possible location on the body, such as arms, legs, torso, etc. Practically, such a scenario represents an unrealistically high user burden and is unfeasible. A related challenge is the seamless integration of wearable electronics in everyday wear items, such as textiles (Heo et al., 2018), clothing, footwear (Hegde et al., 2016), and accessories.

(c) Efficient and informative interpretation of data generated by wearable devices. Wearable devices may generate an abundance of data, for e.g., health-related sensor signals (Redmond et al., 2014). The challenge lies in the interpretation of such data streams and connection with health outcomes, using sensor data to guide behavioral interventions and health education. Emerging methods of artificial intelligence carry a promise of solution to the problem of data analysis and interpretation.

(d) Ultra-low power operation. A wearable device should ideally sustain a lifetime operation without or minimal user interference. In terms of power, this implies operation on a battery, energy harvested from the body, or a combination thereof (Chong et al., 2019; Wang and Daoud, 2019b; Wu et al., 2019). This requires low-power operation both for analog and digital electronics of a wearable. Wireless power delivery (Kim H. et al., 2017) may be explored to seamlessly charge many devices without need to connect each individual device to a charging circuit (for e.g., charging all socks in a drawer), biofuel cells and supercapacitors may need to be utilized in the power subsystems (Bandodkar and Wang, 2016; Dubal et al., 2018).

(e) Flexible and stretchable electronics. Epidermal and body compliant electronic devices (Yeo et al., 2013) may be considered a subset of wearables with additional requirements of allowing shape changes in response to body movement, making such devices especially sensitive to motion artifacts, demanding high biocompatibility and adaptability to variation in human body shapes, sizes, and characteristics (Ray et al., 2019).

(f) Biocompatible communications. Communications from the body (to the outside world) and on the body (between multiple wearables) demand new solutions, as traditional radio methods experience challenges due to absorption by body tissue (Cotton et al., 2014; Paracha et al., 2019). The related challenges include 
development of efficient methods for communicating through or on the body (Seyedi et al., 2013), including the organization of wearables in body sensor networks and their integration into the Internet of Things (Cirani and Picone, 2015).

(g) Biodegradable Electronics. If wearable electronics are to become the true mainstream, the challenge of sustainable, ecologically viable manufacturing, and disposal needs to be addressed (Irimia-Vladu, 2014; Li et al., 2020).

(h) Privacy and Security. By definition, a wearable is an electronic device that resides on or close to a person and is present in a variety of life situations. The challenges include protection of personal information, preventing the unauthorized use of wearables for biometric identification, and ownership of the data produced by wearables (Arias et al., 2015; Cirani and Picone, 2015).

\section{REFERENCES}

Ahmad Tarar, A., Mohammad, U. K., and Srivastava, S. (2020). Wearable skin sensors and their challenges: a review of transdermal, optical, and mechanical sensors. Biosensors 10:56. doi: 10.3390/bios10060056

Ahn, C. R., Lee, S., Sun, C., Jebelli, H., Yang, K., and Choi, B. (2019). Wearable sensing technology applications in construction safety and health. J. Constr. Eng. Manage. 145:03119007. doi: 10.1061/(ASCE)CO.1943-7862.0001708

Arias, O., Wurm, J., Hoang, K., and Jin, Y. (2015). Privacy and security in internet of things and wearable devices. IEEE Trans. Multi Scale Comput. Syst. 1, 99-109. doi: 10.1109/TMSCS.2015.2498605

Atzori, L., Iera, A., and Morabito, G. (2010). The Internet of Things: A survey. Computer Networks 54, 2787-2805. doi: 10.1016/j.comnet.2010.05.010

Bandodkar, A. J., and Wang, J. (2014). Non-invasive wearable electrochemical sensors: a review. Trends Biotechnol. 32, 363-371. doi: 10.1016/j.tibtech.2014.04.005

Bandodkar, A. J., and Wang, J. (2016). Wearable biofuel cells: a review. Electroanalysis 28, 1188-1200. doi: 10.1002/elan.201600019

Bonomi, F., Milito, R., Zhu, J., and Addepalli, S. (2012). "Fog computing and its role in the internet of things," in Proceedings of the First Edition of the MCC Workshop on Mobile Cloud Computing (New York, NY), 13-16.

Chong, Y., Ismail, W., Ko, K., and Lee, C. (2019). Energy harvesting for wearable devices: a review. IEEE Sens. J. 19, 9047-9062. doi: 10.1109/JSEN.2019.2925638

Cirani, S., and Picone, M. (2015). Wearable computing for the internet of things. IT Prof. 17, 35-41. doi: 10.1109/MITP.2015.89

Cotton, S. L., D'errico, R., and Oestges, C. (2014). A review of radio channel models for body centric communications. Radio Sci. 49, 371-388. doi: 10.1002/2013RS005319

Degerli, M., and Ozkan Yildirim, S. (2020). Identifying critical success factors for wearable medical devices: a comprehensive exploration. Univ. Access Inf. Soc. doi: 10.1007/s10209-020-00763-2

Dubal, P. D., Chodankar, N. R., Kim, D.-H., and Gomez-Romero, P. (2018). Towards flexible solid-state supercapacitors for smart and wearable electronics. Chem. Soc. Rev. 47, 2065-2129. doi: 10.1039/C7CS00505A

Gonçalves, C., Ferreira Da Silva, A., Gomes, J., and Simoes, R. (2018). Wearable E-textile technologies: a review on sensors, actuators and control elements. Inventions 3:14. doi: 10.3390/inventions3010014

Hegde, N., Bries, M., and Sazonov, E. (2016). A comparative review of footwearbased wearable systems. Electronics 5:48. doi: 10.3390/electronics5030048

Heo, J. S., Eom, J., Kim, Y.-H., and Park, S. K. (2018). Recent progress of textile-based wearable electronics: a comprehensive review of materials, devices, and applications. Small 14:1703034. doi: 10.1002/smll.20170 3034

\section{CONCLUDING REMARKS}

Wearable electronic technology has immensely stepped into our daily life, being interactive with people in every aspect, and shaping the new lifestyle that technology brings. The development and advances in electronic technology toward further tackling the challenges of wearable devices hold great potential for applications in wearable devices. The impacts on both human conditions and technological properties of the devices need to be considered for realizing interfaces between human and wearable electronics.

\section{AUTHOR CONTRIBUTIONS}

All authors listed have made a substantial, direct and intellectual contribution to the work, and approved it for publication.

Irimia-Vladu, M. (2014). "Green” electronics: biodegradable and biocompatible materials and devices for sustainable future. Chem. Soc. Rev. 43, 588-610. doi: 10.1039/C3CS60235D

Jayathilaka, W. A. D. M., Qi, K., Qin, Y., Chinnappan, A., Serrano-García, W., Baskar, C., et al. (2019). Significance of nanomaterials in wearables: a review on wearable actuators and sensors. Adv. Mater. Weinheim 31:1805921. doi: 10.1002/adma.201805921

Kim, H., Hirayama, H., Kim, S., Han, K. J., Zhang, R., and Choi, J. (2017). Review of near-field wireless power and communication for biomedical applications. IEEE Access 5, 21264-21285. doi: 10.1109/ACCESS.2017. 2757267

Kim, J., Kumar, R., Bandodkar, A. J., and Wang, J. (2017). Advanced materials for printed wearable electrochemical devices: a review. Adv. Electro. Mater. 3:1600260. doi: 10.1002/aelm.201600260

Letocha, C. E., and Dreyfus, J. (2002). Early prints depicting eyeglasses. Arch Ophthalmol. 120, 1577-1580. doi: 10.1001/archopht.120.11.1577

Li, W., Liu, Q., Zhang, Y., Li, C. A., He, Z., Choy, W. C. H., et al. (2020). Biodegradable materials and green processing for green electronics. Adv. Mater. Weinheim 32:2001591. doi: 10.1002/adma.202001591

McCrossen, A. (2013). Marking Modern Times: A History of Clocks, Watches, and Other Timekeepers in American Life. Chicago, IL: University of Chicago Press. doi: 10.7208/chicago/9780226015057.001.0001

Mukhopadhyay, S. C. (2015). Wearable sensors for human activity monitoring: a review. IEEE Sens. J 15, 1321-1330. doi: 10.1109/JSEN.2014.2370945

Nag, A., Mukhopadhyay, S. C., and Kosel, J. (2017). Wearable flexible sensors: a review. IEEE Sens. J 17, 3949-3960. doi: 10.1109/JSEN.2017.2705700

Pacchierotti, C., Sinclair, S., Solazzi, M., Frisoli, A., Hayward, V., and Prattichizzo, D. (2017). Wearable haptic systems for the fingertip and the hand: taxonomy, review, and perspectives. IEEE Trans. Haptics 10, 580-600. doi: 10.1109/TOH.2017.2689006

Paracha, K. N., Rahim, S. K. A., Soh, P. J., and Khalily, M. (2019). Wearable antennas: a review of materials, structures, and innovative features for autonomous communication and sensing. IEEE Access 7, 56694-56712. doi: 10.1109/ACCESS.2019.2909146

Patel, S., Park, H., Bonato, P., Chan, L., and Rodgers, M. (2012). A review of wearable sensors and systems with application in rehabilitation. J. Neuroeng. Rehabil. 9:21. doi: 10.1186/1743-0003-9-21

Rajan, K., Garofalo, E., and Chiolerio, A. (2018). Wearable intrinsically soft, stretchable, flexible devices for memories and computing. Sensors 18:367. doi: $10.3390 / \mathrm{s} 18020367$

Ray, T. R., Choi, J., Bandodkar, A. J., Krishnan, S., Gutruf, P., Tian, L., et al. (2019). Bio-Integrated wearable systems: a comprehensive review. Chem. Rev. 119, 5461-5533. doi: 10.1021/acs.chemrev.8b00573 
Redmond, S. J., Lovell, N. H., Yang, G. Z., Horsch, A., Lukowicz, P., Murrugarra, L., et al. (2014). What does big data mean for wearable sensor systems? Yearb. Med. Inform. 9, 135-142. doi: 10.15265/IY-2014-0019

Seyedi, M., Kibret, B., Lai, D. T. H., and Faulkner, M. (2013). A survey on intrabody communications for body area network applications. IEEE Trans. Biomed. Eng. 60, 2067-2079. doi: 10.1109/TBME.2013.2254714

Shi, Q., Dong, B., He, T., Sun, Z., Zhu, J., Zhang, Z., et al. (2020). Progress in wearable electronics/photonics-moving toward the era of artificial intelligence and internet of things. InfoMat 2, 1131-1162. doi: 10.1002/inf2.12122

Shi, W., Cao, J., Zhang, Q., Li, Y., and Xu, L. (2016). Edge computing: vision and challenges. IEEE Internet Things J. 3, 637-646. doi: 10.1109/JIOT.2016.2579198

Wang, L. Y., and Daoud, W. A. (2019a). Highly flexible and transparent polyionicskin triboelectric nanogenerator for biomechanical motion harvesting. $A d v$ Energy Mater. 9:1803183. doi: 10.1002/aenm.201803183

Wang, L. Y., and Daoud, W. A. (2019b). Hybrid conductive hydrogels for washable human motion energy harvester and self-powered temperature-stress dual sensor. Nano Energy 66:104080. doi: 10.1016/j.nanoen.2019.104080

Witt, D., Kellogg, R., Snyder, M., and Dunn, J. (2019). Windows into human health through wearables data analytics. Curr. Opin. Biomed. Eng. 9, 28-46. doi: 10.1016/j.cobme.2019.01.001

Wu, Y. H., Qu, J. K., Daoud, W. A., Wang, L. Y., and Qi, T. (2019). Flexible composite-nanofiber based piezo-triboelectric nanogenerators for wearable electronics. J. Mater. Chem. A 7, 13347-13355. doi: 10.1039/C9TA02 $345 \mathrm{C}$

Yang, X. Y., and Daoud, W. A. (2016). Triboelectric and piezoelectric effects in a combined tribo-piezoelectric nanogenerator based on an interfacial $\mathrm{ZnO}$ nanostructure. Adv. Funct. Mater. 26, 8194-8201. doi: 10.1002/adfm.2016 02529

Yeo, W.-H., Kim, Y.-S., Lee, J., Ameen, A., Shi, L., Li, M., et al. (2013). Multifunctional epidermal electronics printed directly onto the skin. Adv. Mater. Weinheim 25, 2773-2778. doi: 10.1002/adma.20120 4426

Conflict of Interest: The authors declare that the research was conducted in the absence of any commercial or financial relationships that could be construed as a potential conflict of interest.

Copyright $(\odot 2021$ Sazonov and Daoud. This is an open-access article distributed under the terms of the Creative Commons Attribution License (CC BY). The use, distribution or reproduction in other forums is permitted, provided the original author(s) and the copyright owner(s) are credited and that the original publication in this journal is cited, in accordance with accepted academic practice. No use, distribution or reproduction is permitted which does not comply with these terms. 